Corrigendum

\title{
Corrigendum to "Development of a Patient-Specific Finite Element Model for Predicting Implant Failure in Pelvic Ring Fracture Fixation"
}

\author{
Vickie Shim, ${ }^{1,2}$ Andreas Gather, ${ }^{3}$ Andreas Höch, ${ }^{3}$ David Schreiber, ${ }^{3}$ Ronny Grunert, ${ }^{3}$ \\ Steffen Peldschus, ${ }^{4}$ Christoph Josten, ${ }^{3}$ and Jörg Böhme ${ }^{3}$ \\ ${ }^{1}$ Auckland Bioengineering Institute, University of Auckland, 70 Symonds Street, Auckland, New Zealand \\ ${ }^{2}$ Menzies Health Institute, Griffith University, Gold Coast, QLD, Australia \\ ${ }^{3}$ Department of Trauma, Plastic and Reconstructive Surgery, University of Leipzig, Liebigstr. 20, 04103 Leipzig, Germany \\ ${ }_{4}^{4}$ Institute of Forensic Medicine, Ludwig-Maximilians-University Munich, Munich, Germany
}

Correspondence should be addressed to Jörg Böhme; joerg.boehme@medizin.uni-leipzig.de

Received 1 November 2017; Accepted 7 November 2017; Published 23 November 2017

Copyright ( 2017 Vickie Shim et al. This is an open access article distributed under the Creative Commons Attribution License, which permits unrestricted use, distribution, and reproduction in any medium, provided the original work is properly cited.

In the article titled "Development of a Patient-Specific Finite Element Model for Predicting Implant Failure in Pelvic Ring Fracture Fixation" [1], Dr. Andreas Gather, Mr. David Schreiber, and Dr. Christoph Josten were missing from the authors' list. The corrected authors' list is shown above.

\section{References}

[1] V. Shim, A. Höch, R. Grunert, S. Peldschus, and J. Böhme, "Development of a patient-specific finite element model for predicting implant failure in pelvic ring fracture fixation," Computational and Mathematical Methods in Medicine, vol. 2017, Article ID 9403821, 11 pages, 2017. 


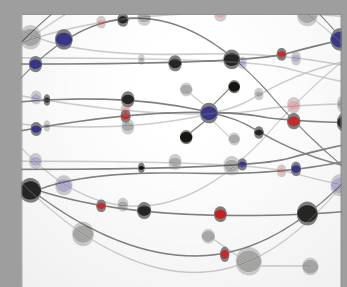

The Scientific World Journal
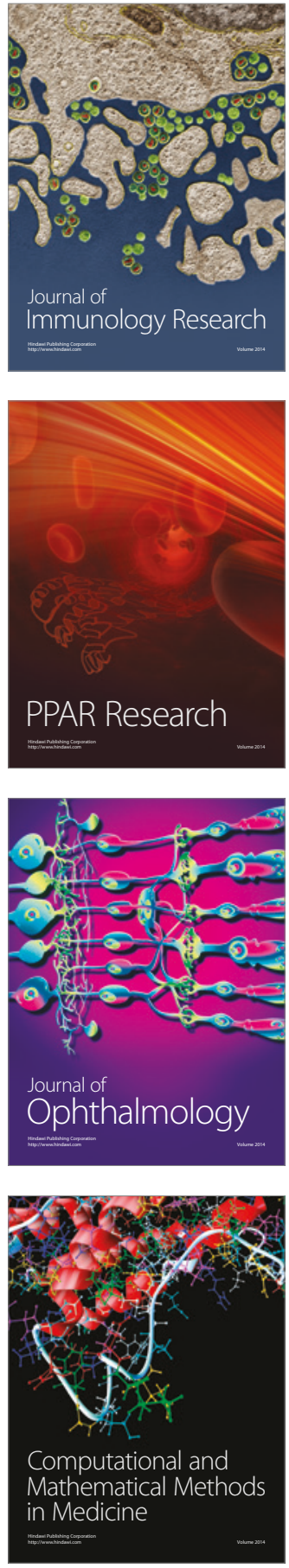

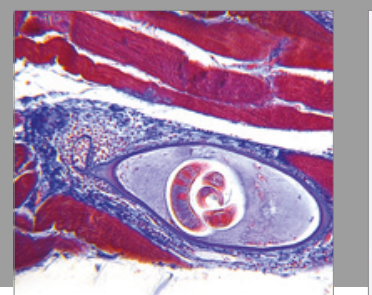

Gastroenterology Research and Practice
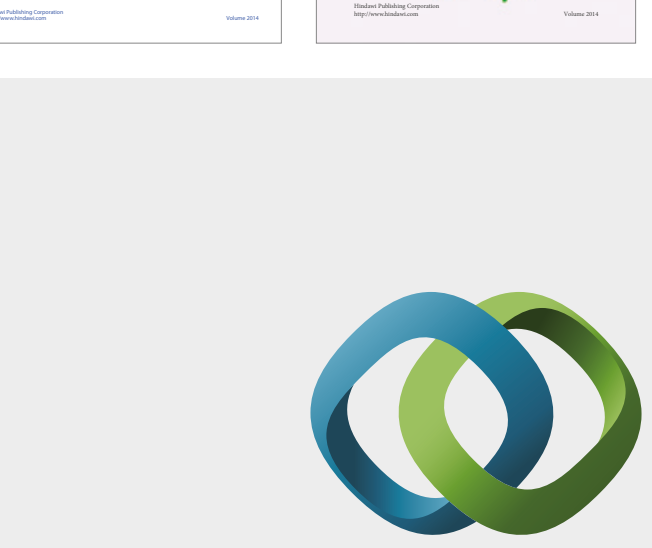

\section{Hindawi}

Submit your manuscripts at

https://www.hindawi.com
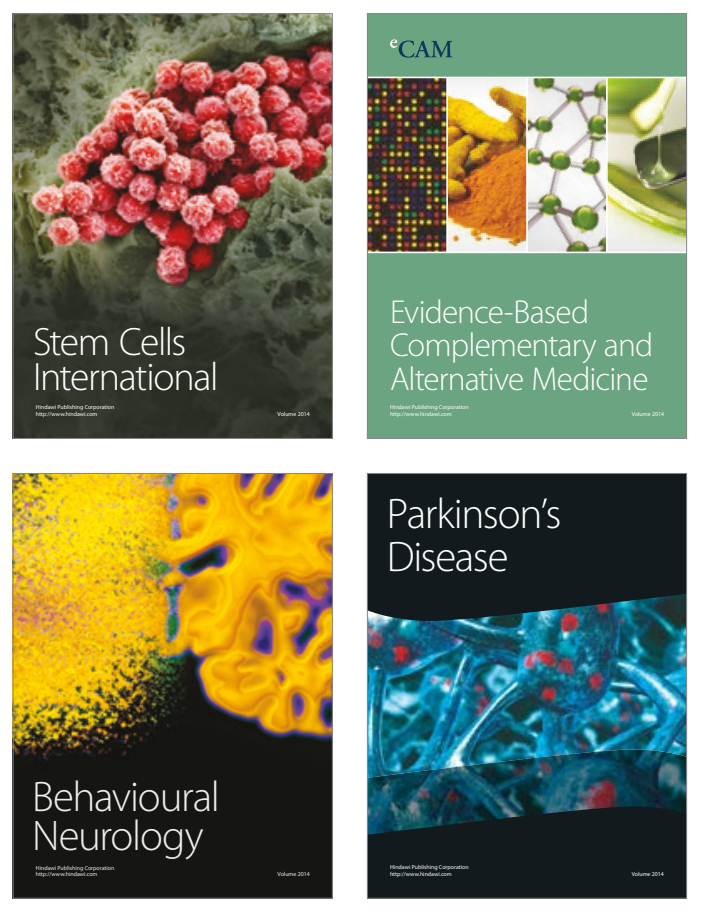
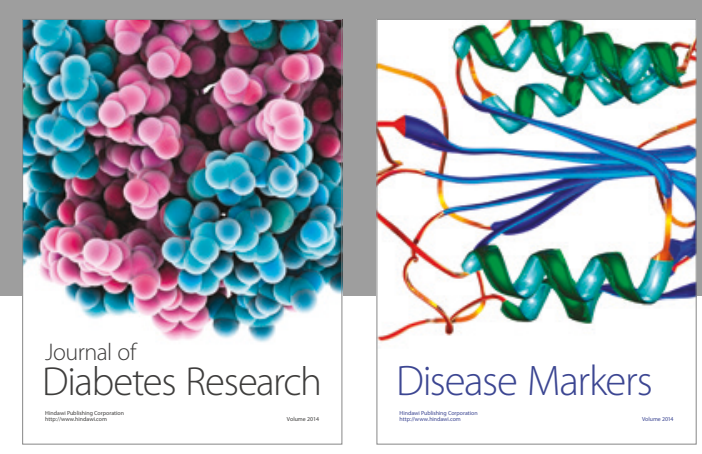

Disease Markers
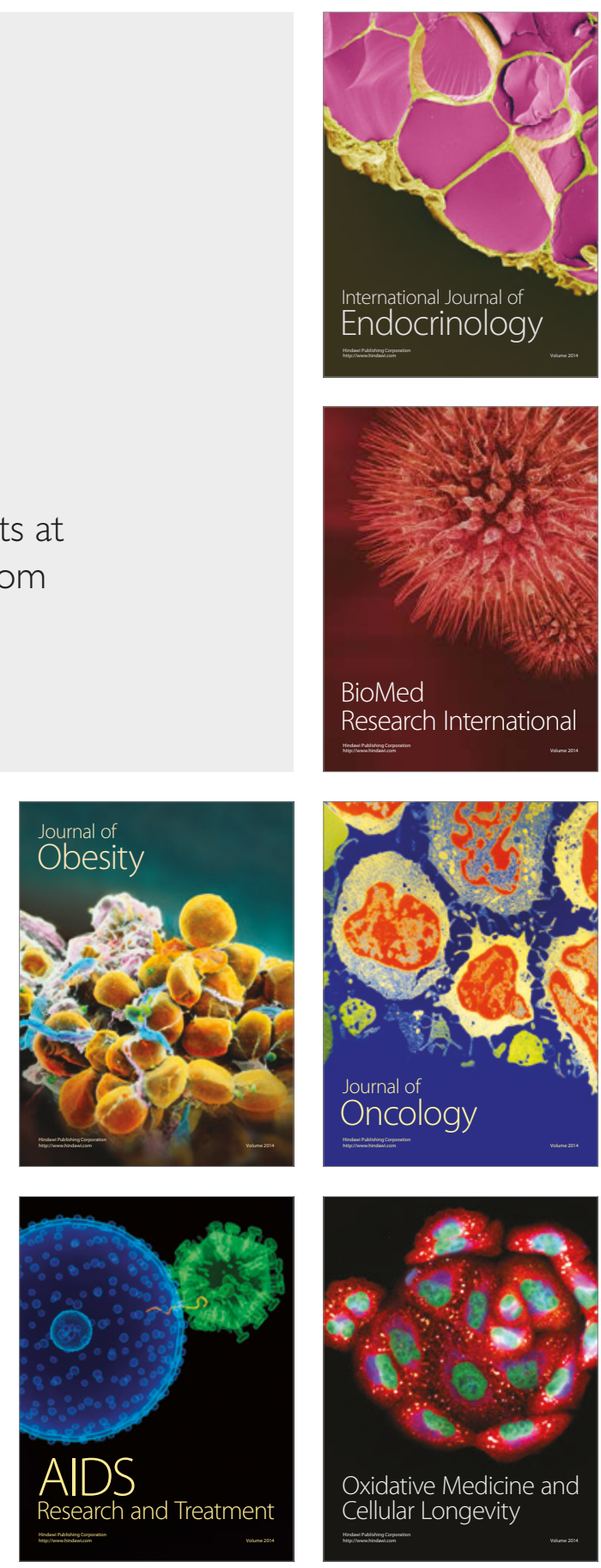